\title{
Large quantities of low molecular weight IgM in mixed cryoglobulinaemia
}

\author{
P J ROBERTS-THOMSON, ALISON KENNEDY, AND LIN Y KOH
}

From the Department of Clinical Immunology, Flinders Medical Centre, Bedford Park, South Australia 5042

SUMmary Low molecular weight (LMW) IgM is the monomeric subunit of pentameric IgM. It was not found in the sera of 20 healthy subjects but was detected in all six patients with mixed cryoglobulinaemia with a mean value of $1.4 \mathrm{~g} / \mathrm{l}$, representing $34 \%$ of the total IgM. In three of four patients studied LMW IgM was monoclonal and of the same light chain type $(x)$ as the pentameric monoclonal IgM rheumatoid factor (RF) observed in the cryoprecipitate. LMW IgM was proportionately under-represented, however, in the cryoprecipitate compared with the corresponding serum, possibly because of the lower valency of the LMW molecule. Immunoblot analysis of sera showed the presence of other oligomers of IgM in addition to monomeric IgM, suggesting that a disorder of IgM assembly was responsible for its occurrence, and this was supported by the secretion of large proportions of LMW IgM in vitro by peripheral blood mononuclear cells (PBMC) from one patient with this disorder but not from healthy controls. In conclusion, the occurrence of large quantities of monoclonal LMW IgM in mixed cryoglobulinaemia was observed, and it is suggested that this is unlikely to have a direct pathogenic signifiance. It is postulated that its presence reflects a disturbance of assembly of the monomeric IgM subunits that occurs during the polymerisation of the pentameric molecule.

Key words: rheumatoid factor, IgM synthesis.

Mixed cryoglobulinaemia is an uncommon clinical syndrome that consists of polyarthralgia, myalgia, recurrent palpable purpura and, in many instances, nephritis. ${ }^{1}$ The basic pathological feature is the presence of a necrotising vasculitis occurring particularly in the skin and kidney. Mixed cryoglobulinaemia may occur as an isolated clinical entitynamely, the essential or idiopathic form, or in association with lymphoproliferative, infective, or autoimmune rheumatic disorders. The cause is unknown, though some patients have evidence of past or present infection with hepatitis B virus. ${ }^{2}$

A large variety of immunological abnormalities have been reported in this condition particularly involving the humoral arm of the immune response. ${ }^{3}$ These include the presence of large quantities of mixed cryoglobulins, consisting in most cases of monoclonal $\varkappa$ IgM with RF activity and polyclonal IgG; low levels of haemolytic comple-

Accepted for publication 10 July 1987.

Correspondence to Dr P J Roberts-Thomson, Department of Clinical Immunology, Flinders Medical Centre, Bedford Park, South Australia 5042. ment, $\mathrm{C} 3$ and $\mathrm{C} 4$, and high levels of serum RF and circulating immune complexes. Serum immunoglobulins may be increased, normal, or depressed, and raised titres to various ubiquitous viruses are described. ${ }^{4}$ In the current study we report a further humoral abnormality in this disorder-namely, the occurrence of considerable quantities of LMW IgM which appear to have a monoclonal origin in most cases. Furthermore, we present data suggesting that the LMW IgM arises owing to a disorder of assembly of the IgM subunits during IgM synthesis.

\section{Patients and methods}

PATIENTS AND CONTROLS

Six patients with mixed cryoglobulinaemia (four female and two male) with a mean age of 69 years were studied. Five patients had essential mixed cryoglobulinaemia and one patient had mixed cryoglobulinaemia secondary to a lymphoproliferative disorder (patient 1, Table 1). The diagnosis of essential mixed cryoglobulinaemia was made according to standard clinical, laboratory, and histopathological criteria. ${ }^{5}$ Control sera were 
obtained from 20 healthy laboratory personnel. All sera were separated from venous blood after clotting at $37^{\circ} \mathrm{C}$ and were stored at $-80^{\circ} \mathrm{C}$.

ISOLATION OF CRYOGLOBULIN

Serum was allowed to stand at $4^{\circ} \mathrm{C}$ for 72 hours, and the cryoglobulin was separated by centrifugation at $1000 \mathrm{~g}$ for 20 minutes at $4^{\circ} \mathrm{C}$. The cryoglobulin was then dissolved in warmed $\left(37^{\circ} \mathrm{C}\right)$ saline and the process of cryoprecipitation repeated over 72 hours. The cryoglobulin was then washed twice with iced saline.

DETECTION AND MEASUREMENT OF LMW IgM LMW IgM was detected in sera by two methods.

\section{Filtration chromatography}

A filtration chromatographic method was used, as described by Roberts-Thomson et al. ${ }^{6}$ In brief, serum diluted in equal volumes of $0 \cdot 1 \mathrm{M} \mathrm{Na}$ acetate buffer, $\mathrm{pH} 4 \cdot 0$, was separated at room temperature on Sepharose 6B or Sephacryl S300 (Pharmacia, Uppsala, Sweden), equilibrated in phosphate

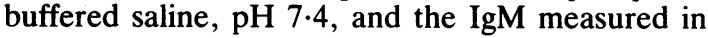
the eluate fraction by laser nephelometry or enzyme linked immunosorbent assay (ELISA). ${ }^{7}$ An IgM profile was then obtained and the detection of a second IgM peak eluting just before the IgG peak, signified the presence of LMW IgM. Planimetry was used to determine the quantity of LMW IgM.

\section{Immunoblot analysis}

The method of Harries et $a l^{8}$ was used. In brief, serum was separated on sodium dodecyl sulphate/ $3.6 \%$ polyacrylamide gel slabs and the separated serum proteins transferred to nitrocellulose. The IgM bands were developed using a biotin-avidin antihuman $\mu$ chain specific system. Serum with and without LMW IgM were included in each run as controls.

OTHER SEROLOGICAL INVESTIGATIONS $C$ reactive protein (CRP), serum immunoglobulins, and RF were measured by rate nephelometry (Beckmans ICS). Circulating immune complexes (CIC) were measured by the liquid phase Clq binding assay of Zubler et al. ${ }^{9}$ Measurement of cryoprotein from $1 \mathrm{ml}$ of serum was by the ponceau $S$ dye binding method and identification of its specific proteins by zone electrophoresis and immunoelectrophoresis using commercially derived antisera (Dakopatts, Glostrup, Denmark).

SECRETION OF LMW IgM IN VITRO

Peripheral blood mononuclear cells were separated from heparinised blood by Ficoll-Hypaque sedi-

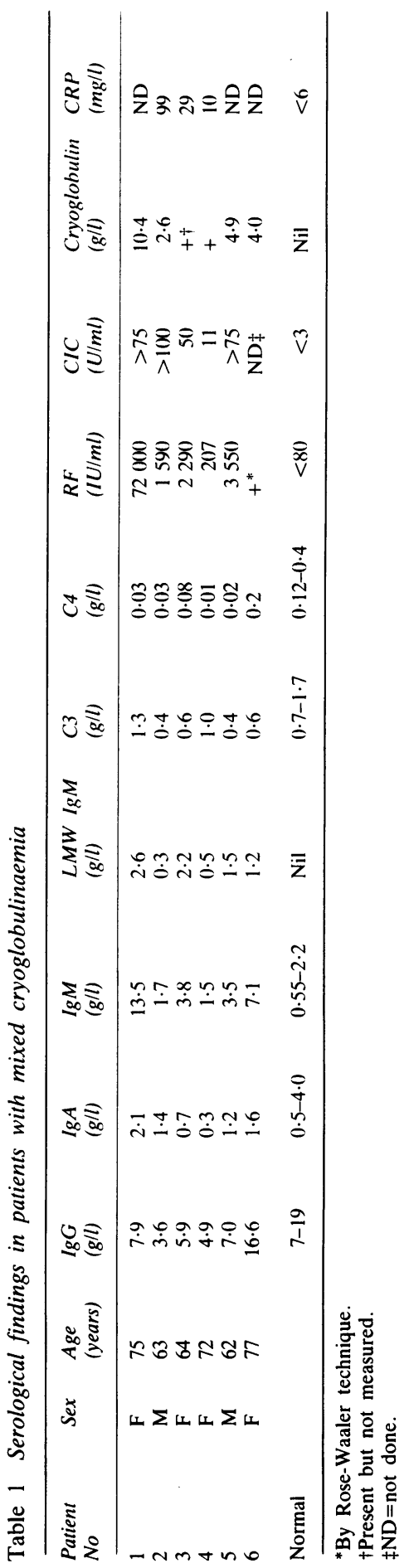


mentation using standard techniques. No patient was receiving cytotoxic chemotherapy at the time of study. Replicate $2 \mathrm{ml}$ cultures were established containing $2 \times 10^{6} \mathrm{PBMC}$ and pokeweed mitogen at a final concentration of $1 \mu \mathrm{g} / \mathrm{ml}$. The medium was changed at day 3 . At day 7 the culture supernatants were collected (total volume $15-20 \mathrm{ml}$ ), concentrated in an Amicon concentrator unit to a final volume of $0.5 \mathrm{ml}$, and applied to a $90 \times 1.6 \mathrm{~cm}$ Sephacryl S300 column. Fractions $(4 \mathrm{ml})$ were collected and assayed for IgM using a specific IgM ELISA as described elsewhere. ${ }^{7}$ The lower limit of sensitivity of this assay for IgM is $20 \mathrm{ng} / \mathrm{ml}$. To confirm active synthesis and secretion of $\operatorname{IgM}$ in vitro, cycloheximide was added to some of the culture to a final concentration of $10 \mu \mathrm{g} / \mathrm{ml}$.

\section{Results}

Serological findings for the six patients are shown in Table 1. Considerable quantities of mixed cryoglobulins were found in all patients, and the properties of these are shown in Table 2. All patients' sera contained LMW IgM (mean level $1.4 \mathrm{~g} / \mathrm{l}$ ), which contributed between 16 and $59 \%$ of the total IgM, but LMW IgM was not detected in any of the control sera. In three patients the proportion of high molecular weight (HMW) and LMW IgM in the supernatant after cryoprecipitation was compared with the proportion in the original serum (Table 3, Fig. 1). An increase in the proportion of LMW IgM was seen in the supernatant, suggesting that the HMW IgM was preferentially represented in the cryoprecipitate.

Column fractions containing LMW IgM from four patients were concentrated and analysed by zone and immunoelectrophoresis. In three patients the LMW IgM was identified as monoclonal $\operatorname{IgM} x$, whereas in the fourth it appeared to be polyclonal with both $x$ and $\lambda$ light chain types.

Serum samples, cryoprecipitates, and serum

Table 2 Characterisation of mixed cryoglobulins

\begin{tabular}{|c|c|c|c|}
\hline $\begin{array}{l}\text { Patient } \\
\text { No }\end{array}$ & $\begin{array}{l}\text { Cryoglobulin } \\
(g / l)^{*}\end{array}$ & $\begin{array}{l}\text { Zone and } \\
\text { immunoelectrophoretic } \\
\text { appearance }\end{array}$ & $\begin{array}{l}R F \\
\text { activity }\end{array}$ \\
\hline 1 & $10 \cdot 4$ & $m \dagger \operatorname{IgM} x+p+\operatorname{IgG}$ & + \\
\hline 2 & $2 \cdot 6$ & $m \operatorname{IgM} x+p \operatorname{IgG}$ & + \\
\hline 3 & $\therefore \neq$ & $m \operatorname{IgM} x+p \operatorname{IgG}$ & + \\
\hline 4 & + & p $\operatorname{IgM}+p \operatorname{IgG}$ & + \\
\hline 5 & $4 \cdot 9$ & $m \operatorname{IgM} x+p \operatorname{IgG}$ & + \\
\hline 6 & $4 \cdot 0$ & Not done & + \\
\hline
\end{tabular}

${ }^{*}$ Derived from $1 \mathrm{ml}$ of serum.

$+\mathrm{m}=$ monoclonal; $\mathrm{p}=$ polyclonal

$\ddagger$ Detected but not measured. supernatants from five patients were also analysed by immunoblotting using IgM specific antisera (Fig. 2). In addition to the identification of a band representing the monomer (mol. wt approximately

Table 3 Proportion of IgM occurring as the low molecular weight moiety in original serum and in supernatant after cryoprecipitation

\begin{tabular}{lll}
\hline Patient & \multicolumn{2}{l}{ Percentage } \\
No & OMW IgM \\
\cline { 2 - 3 } & Original serum & Supernatant \\
\hline 1 & $15 \cdot 9$ & $19 \cdot 6$ \\
2 & $17 \cdot 0$ & $45 \cdot 0$ \\
4 & $35 \cdot 0$ & $48 \cdot 0$ \\
\hline
\end{tabular}

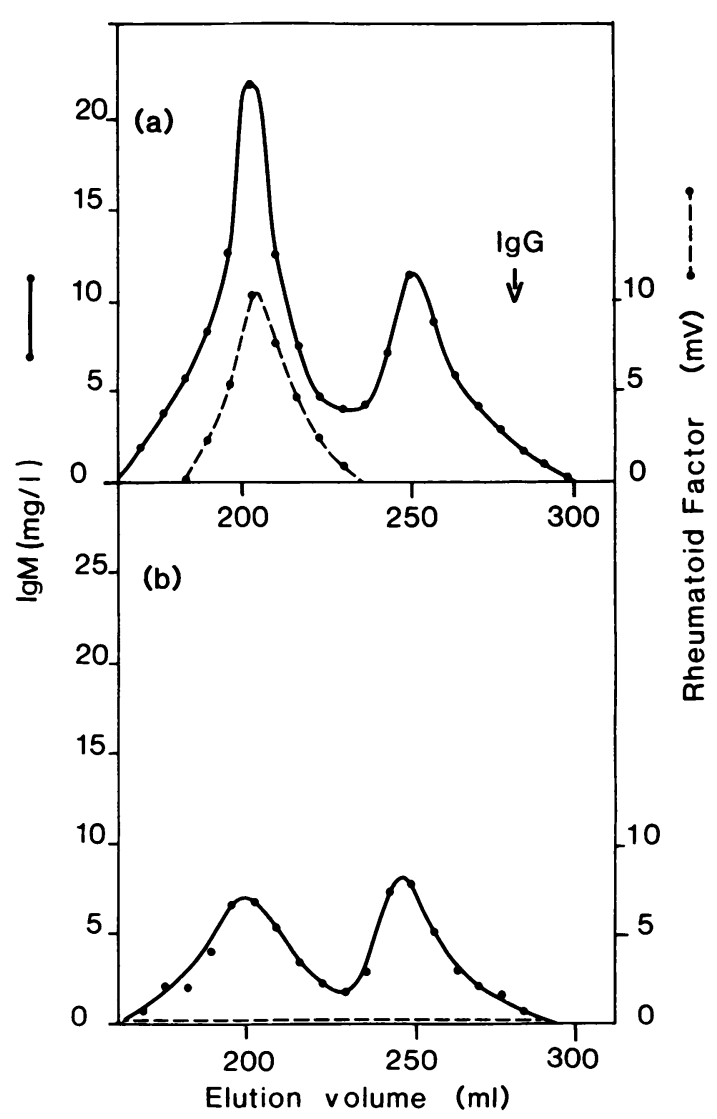

Fig. 1 Elution profile of IgM and RF activity after Sepharose $6 B$ chromatography of $(a)$ the original serum of patient No 3 (Table l) and (b) the corresponding serum supernatant after separation of cryoprecipitate. Note the loss of $R F$ activity in the supernatant and the relative preservation of the $L M W \operatorname{IgM}$. RF is measured by a nephelometric technique and expressed in millivolts $(\mathrm{mV}) . "$ 


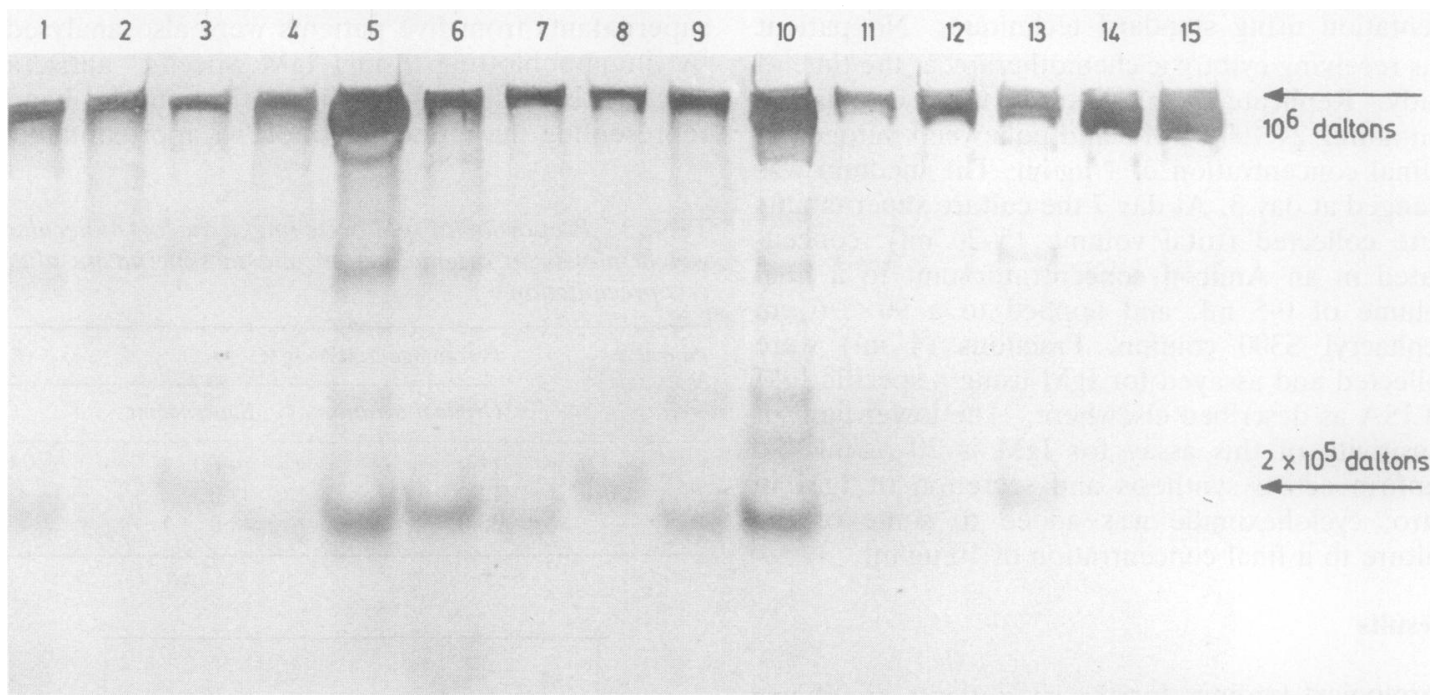

Fig. 2 Immunoblot appearance of original sera (lanes 1-5) and corresponding supernatants (lanes 6-10) and cryoprecipitates (lanes 11-15) stained with an anti $\mu$-avidin, biotin, avidin-alkaline phosphatase, substrate sandwich. In normal serum (not shown) only pentameric IgM bands are observed.

$2 \times 10^{5}$ daltons) in all samples (although less evident in the cryoprecipitate specimens), other higher molecular weight bands were also identified migrating at the dimer and trimer positions and also intermediary bands between the monomer and dimer positions. The molecular nature of these intermediary bands was not determined. In control sera these lower molecular weight IgM bands were not seen and only pentameric bands were observed.

PBMC from two patients and three controls were stimulated with pokeweed mitogen and cultured over seven days. The supernatants were then concentrated and analysed for LMW IgM by filtration chromatography. LMW IgM was not detected in any of the controls but was found in large proportions $(80 \%$ of the total secreted $\operatorname{IgM})$ in the supernatant from pokeweed stimulated PBMC from one of the patients. Insufficient IgM was secreted in vitro in the second patient to allow satisfactory chromatographic analysis. Cycloheximide inhibited IgM secretion by more than $90 \%$.

\section{Discussion}

Large quantities of predominantly monoclonal LMW IgM have been identified in the sera of all six patients with mixed cryoglobulinaemia studied. In three of four patients studied the LMW IgM was monoclonal and, as the light chain type was identical to the monoclonal pentameric IgM observed in the cryoglobulin, this association suggests that the origi $\infty$ of the LMW IgM was from the same clone of lymphoid cells that was secreting the pentameneo IgM. In another study, using a specific RF radioimmunoassay, we found that partially purified LMW IgM obtained from patient 1 of this current $\frac{\circ}{\mathrm{D}}$ study had RF activity, and this was also observed for LMW IgM prepared from another patient with $\overrightarrow{\overrightarrow{0}}$ mixed cryoglobulinaemia (not included in the present study). ${ }^{10}$ In this study it was not possible to test the LMW IgM fractions from the remaining patients for RF activity. When all the above observations are considered it appears likely that $\frac{0}{-}$ most of the LMW IgM found in mixed cryoglobulin- 3 aemia is derived from the same clone that secretes the pentameric IgM RF which participates in the cryoglobulin.

The clinical significance of the circulating $\mathrm{LMW}^{\mathrm{S}}$ IgM found in mixed cryoglobulinaemia is uncertain. Owing to its divalent or monovalent nature ${ }^{11}$ it has far less avidity than its corresponding pentameric $\tilde{N}$ pentavalent member. This is a possible explanation N for its under-representation in the cryoprecipitate, N which consists predominantly of IgM RF, and its ${ }^{\omega}$ corresponding antigen-namely, polyclonal IgG. If one assumes that this immune complex has pathological consequences it would appear that LMW IgM because of its lower valence would have less ${ }_{D}$ pathogenic potential than the pentameric molecule. ${ }_{0}^{\circ}$ In addition, we have observed persistently high levels of LMW IgM in two of our patients in the $\frac{?}{\mathbb{Q}}$ 
absence of disease activity, suggesting that the LMW IgM in itself probably has little pathogenic significance but is a marker of the clonal expansion which appears to be a fundamental abnormality in this disorder.

The reasons for the secretion of LMW IgM are unclear. As previously discussed, ${ }^{12}$ several hypotheses can be proposed. We feel the most likely explanation for the occurrence of LMW IgM is a defect in the assembly of monomeric IgM subunits during the polymerisation of the pentameric molecule. This hypothesis would be in accordance with the observed secretion of LMW IgM from the PMBC from one patient but not controls and with the finding of additional oligomers and other intermediary molecular forms of IgM in the sera of our patients. Whether this defect is related to a disorder of $J$ chain availability or function, or both, or to deficiencies in the probable polymerising enzyme sulphydryl oxidase ${ }^{13}$ is unclear.

In conclusion, we have observed large quantities of monomeric and oligomeric IgM in patients with mixed cryoglobulinaemia. In most patients this LMW IgM appeared to be monoclonal in origin and of the same light chain type as the pentameric IgM $\mathrm{RF}$ found in cryoglobulin. In a separate study we have identified RF activity in the LMW IgM fraction. The reason for the secretion of LMW IgM in this disorder is unknown, but we postulate a disorder of assembly of monomeric IgM subunits during polymerisation of the pentameric molecule.

We thank Wendy Graham and Mary Brown for typing the script and helpful secretarial assistance. Drs $A$ Bune and $N$ Hurst for allowing us to study their patients, and the NH \& MRC and Arthritis Foundation of Australia (SA) for financial assistance.

\section{References}

1 Meltzer M, Elias K, McCluskey R T, Cooper N, Franklin E C. Cryoglobulinemia-a clinical and laboratory study. Am J Med 1966; 40: 837-56.

2 Levo Y, Gorevic P D, Kassab H J, Zucker-Franklin D, Franklin E C. The association between hepatitis B virus and cssential mixed cryoglobulinemia. $N$ Engl J Med 1977; 296: $1501-4$.

3 Brouet J C, Clauvel J P, Danon F, Kleim M. Seligmann N. Biological and clinical significance of cryoglobulins. Am J Med 1974; 57: 775-88.

4 Fiorini G, Bernasconi P, Sinico R A. Chiancse R, Pozzi F, D'Amico G. Increased frequency of antibodies to ubiquitous viruses in essential mixed cryoglobulinaemia. Clin Exp Immunol 1986; 64: 65-70.

5 Gorevic P D, Kassab H J, Levo Y, et al. Mixed cryoglobulinemia: clinical aspect and long term follow-up of 40 patients. Am J Med 1980; 69: 287-308.

6 Roberts-Thomson P J, Ncoh S H, Bradlcy J. Quantitation and evaluation of low molecular weight IgM in rheumatoid arthritis. Ann Rheum Dis 1980; 39: 349-53.

7 Jones D N, Koh L Y, Bertouch J V, Roberts-Thomson P J. Synthesis of low molecular weight IgM in vitro in rheumatoid arthritis. Arthritis Rheum 1985; 28: 112-8.

8 Harries R, Beckman I, Roberts-Thomson P J. Low molccular weight IgM: detection using immunoblotting. J Immunol Methods 1986; 88: 97-100.

9 Zubler R H, Nydegger V, Perrin L H, et al. Circulating and intra-articular immunc complexes in paticnts with rhcumatoid arthritis. Corrclation of ${ }^{125} \mathrm{I} \mathrm{Clq}$ binding activity with clinical and biological features of the discasc. J Clin Invest 1976; 57: 1308-19.

10 Roberts-Thomson P J. Wcrnick R M. Ziff M. Low molccular weight IgM in rhcumatoid arthritis and other rheumatic discascs. Arthritis Rheum 1981; 24: 795-802.

11 Chavin S I, Franklin E C. Studies on antigen binding activity of macroglobulin antibody subunits and their enzymatic fragments. J Biol Chem 1969; 244: 1345-52.

12 Koh L Y, Joncs D N, Roberts-Thomson P J. Appcarance of low molccular weight IgM during course of infective endocarditis. Clin Exp Immunol 1986; 64: 471-5.

13 Roth R A, Koshland M E. Identification of a lymphocytc enzyme that catalyzes pentamer immunoglobulin $M$ assembly. $J$ Biol Chem 1981; 256: 4633-9. 